REVIEW

\title{
Critical care in the emergency department: shock and circulatory support
}

\author{
C A Graham, T R J Parke
}

Emerg Med J 2005;22:17-21. doi: 10.1136/emj.2003.012450

Effective resuscitation includes the rapid identification and correction of an inadequate circulation. Shock is said to be present when systemic hypoperfusion results in severe dysfunction of the vital organs. The finding of normal haemodynamic parameters, for example blood pressure, does not exclude shock in itself. This paper reviews the pathophysiology, resuscitation, and continuing management of the patient presenting with shock to the emergency department.

See end of article for authors' affiliations

.....................

Correspondence to: Dr T R J Parke, Accident and Emergency

Department, Southern General Hospital, Glasgow G51 4TF, UK. tim.parke@sgh.scot.nhs.uk

Accepted for publication 8 April 2004

\section{CLINICAL SCENARIO}

A 61 year old man is admitted to the resuscitation room with shortness of breath. He has a medical history of ischaemic heart disease treated with quadruple coronary artery bypass grafting five years ago. He has been unwell for the past 48 hours with a productive cough, lethargy, and fever.

Vital signs on arrival include temperature of $35.2^{\circ} \mathrm{C}$, respiratory rate $40 / \mathrm{min}$, pulse 130 beats/ min, non-invasive blood pressure $70 / 40 \mathrm{~mm} \mathrm{Hg}$, and oxygen saturation of $90 \%$ on $15 \mathrm{l} / \mathrm{min}$ face mask oxygen using a non-rebreather bag. He is poorly perfused, cold, and shut down. Initial blood gas analysis shows a mixed respiratory and metabolic acidosis.

\section{Questions}

- Is this patient suffering from shock?

- What is the pathophysiology of the shock process?

- What should the initial management in the first 15 minutes be for this patient?

\section{PHYSIOLOGY}

Shock is defined as acute circulatory failure with

will produce organ failure and, in the context of global hypoperfusion, multiple organ failure ensues. The aim of resuscitation is to prevent shock worsening and to restore the circulation to a level that meets the body's tissue oxygen requirements.

Shock can arise through a variety of mechanisms. Pump failure may be attributable to inadequate preload (for example, severe bleeding), myocardial failure, or excessive afterload. Shock can also occur with an adequate or increased cardiac output as seen in distributive shock (for example, septic or anaphylactic shock). Vital tissues remain ischaemic as much of the cardiac output is distributed inappropriately through vascular beds.

The mechanisms producing shock may also be very complex involving combinations of these factors. For example, in septic shock, there may be reduced preload from increased vascular permeability and venodilatation, impaired myocardial contractility caused by inflammatory mediators, and tissue hypoxia from inappropriate distribution of blood flow.

Compensatory mechanisms are activated in response to tissue hypoperfusion. Acutely, the adrenergic autonomic nervous system is activated. Venoconstriction increases the preload to the heart; vasoconstriction of all non-essential arterial beds sustains arterial blood pressure and myocardial contractility is maximised. This response attempts to optimise cardiac output and maintain an adequate perfusion pressure to the coronary arteries and brain. Thus, the early stages of uncomplicated shock are characterised by tachycardia and a comparatively normal blood pressure.

Further compensatory neuroendocrine mechanisms are activated in the kidney. This delayed effect consists of rennin-angiotensin-aldosterone mediated renal retention of salt and water to further maximise preload and improve cardiac output.

Hypotension occurs once the compensatory mechanisms have been overwhelmed, or the autonomic nervous system is unable to respond effectively. Reasons for this include advanced age, concurrent medication (for example $\beta$ block), autonomic neuropathy, or adrenal insufficiency.

In profound shock other autonomic mechanisms, primarily vagal, may come into play. In the presence of low cardiac filling pressures in severe hypovolaemia, the tachycardia response to shock

In early shock a switch from aerobic to anaerobic metabolism compensates temporarily. Lactate accumulates as a result of this anaerobic metabolism. Hypoxia eventually causes cellular and then tissue necrosis. Loss of sufficient tissue
Abbreviations: ED, emergency department; IV, intravenous; ECG, electrocardiography; CVP, central venous pressure 
may be replaced with a reflex bradycardia. As shock progresses, cells in ischaemic tissues switch to anaerobic metabolism and lactic acidosis stimulates compensatory hyperventilation.

Identification and treatment of shock in the emergency department is based on sound knowledge of these physiological principles.

\section{CLINICAL FEATURES OF SHOCK}

Clinical features of acute circulatory failure are usually those of tissue hypoperfusion. This is most easily detected in the skin as central pallor, peripheral cyanosis, and sluggish capillary return. Other clinical evidence could include a raised respiratory rate, confusion, or coma. Renal hypoperfusion is indicated by a diminished urine output. Cardiac ischaemia may be manifest on electrocardiographic monitoring. Arterial blood gas analysis may show a metabolic (lactic) acidosis.

The traditional vital signs are less reliable indicators of shock. The interplay between the sympathetic and parasympathetic autonomic nervous system can produce pulse rates and blood pressures that are normal, high, or low. Shock cannot be excluded solely on the basis of normal vital signs.

Blood sugar measurement can identify patients with shock secondary to hyperglycaemia (diabetic ketoacidosis and hyperosmolar states) and with hypoglycaemia, which may resemble shock clinically.

\section{INITIAL MANAGEMENT OF SHOCK IN THE EMERGENCY DEPARTMENT}

Ideally, patients suffering from shock are identified at triage and transferred to the resuscitation room. All patients should be given high flow oxygen, have intravenous (IV) access secured, and have basic monitoring instituted (non-invasive blood pressure, pulse oximetry, and continuous ECG).

\section{MANAGEMENT OF AIRWAY AND BREATHING} Does the patient require intubation and ventilation? Consider early intubation and ventilation for severe shock if there is respiratory distress, severe hypoxaemia, pronounced acidosis, or coma. Intubation ensures protection from aspiration in the presence of a reduced conscious level. Where agitation is attributable to cerebral hypoxia, intubation and ventilation permits rapid treatment without precipitating further respiratory compromise. Inspired oxygen can be maximised to $100 \%$ to optimise oxygen delivery to the tissues. Finally, the increased work of breathing with its resultant oxygen requirements is removed.

Profoundly shocked patients, particularly with severe acidosis or impaired conscious level, should be intubated and ventilated within 15 minutes of arrival in the resuscitation room. Senior anaesthetic and intensive care input should be sought at an early stage. Induction carries particular risks in the presence of severe shock, with a significant chance of precipitating profound circulatory collapse through the myocardial depressant effects and vasodilating properties of many induction agents.

A rapid sequence intubation technique is required using comparatively cardio-stable agents such as etomidate or ketamine. Modified doses will be needed, particularly in the presence of hypotension. Pre-loading the circulation with IV fluids to correct hypovolaemia may be necessary, and the use of pressor agents may be urgently required. Low tidal volumes and peak inspiratory pressures should be the aim immediately after intubation to prevent the reduction in venous return that is associated with positive pressure ventilation.

\section{INITIAL MANAGEMENT OF THE CIRCULATION}

\section{Is a fluid challenge indicated?}

This can be a difficult decision to make on clinical grounds and often depends on the context in which the patient has presented. Conditions that are associated with actual or relative hypovolaemia respond well to restoration of vascular volume. Such conditions should be identified early. Blood loss can be assumed to be the cause of shock after trauma, at least initially, while a search is carried out for haemorrhage into the chest, pelvis, abdomen, or externally.

In conditions such as diabetic ketoacidosis, bowel obstruction, or severe diarrhoea and vomiting, it is reasonable to assume initially that salt and water depletion have caused hypovolaemic shock.

If it is clear that shock has been caused by hypovolaemia, IV fluids should be started. Patients should receive an initial bolus of one to two litres of IV fluid rapidly and be reassessed. The choice of fluid remains controversial, but crystalloids, particularly Ringer's lactate, have become widely supported. ${ }^{1}$ There is no place for inotropes in the management of severe hypovolaemia unless the patient is in established cardiac arrest, as they may precipitate severe arrhythmias that may in turn worsen the shock state.

In other circumstances fluid therapy can be harmful. A patient with acute myocardial infarction or compromising arrhythmia may progress to cardiogenic shock with left ventricular failure and pulmonary oedema. Such patients may be identified by a previous cardiac history, recent chest pain, or ECG changes together with the clinical and radiological signs of acute pulmonary oedema. Additional fluid loading in these patients may increase an already increased left ventricular end diastolic pressure and worsen pulmonary oedema with no useful gain in terms of cardiac output. This is attributable to the flat nature of the Starling curve in the failing heart. Initial mangement in these circumstances is targeted at immediate treatment of any arrhythmia and early inotropic support, together with aggressive management of pulmonary oedema.

A second scenario in which fluids may be harmful is where there is severe shock associated with ongoing noncompressible haemorrhage, for example, penetrating trauma to the torso or a leaking aortic aneurysm. There is evidence that large volume resuscitation before surgical control of haemorrhage is harmful ${ }^{2}$ and these patients should be treated using the principles of hypotensive resuscitation. This approach has been shown to lead to fewer complications such as dilutional coagulopathy, hypothermia, and postoperative adult respiratory distress syndrome. ${ }^{2}$

It entails giving minimal, ideally no, IV fluid to the patient until they are in the operating room and at the point of surgical control of haemorrhage. Enough fluid is given to maintain consciousness (brain perfusion). Blood is the colloid of choice if significant blood loss is suspected.

Hypotensive resuscitation is not recommended, however in the context of blunt multiple trauma and where there is evidence of serious head injury, a systolic pressure of at least $90 \mathrm{~mm} \mathrm{Hg}$ must be maintained. ${ }^{34}$

\section{Should we give a fluid bolus in shock of uncertain aetiology?}

If anaphylactic shock is suspected (rash, wheeze, allergen exposure), then fluid therapy is appropriate along with intramuscular adrenaline (epinephrine). ${ }^{5-7}$ Similarly, if septic shock is suspected (petechial rash, high fever, presence of infective source, rigid abdomen), then fluids should be given. $^{89}$ In addition to maldistribution, septic shock also has a large hypovolaemic component because of the extravasation of plasma through the leaking vasculature. ${ }^{89}$ 
Occasionally a patient will present with shock with no immediate obvious precipitant. The usual causes are occult haemorrhage (such as upper gastrointestinal haemorrhage without haemetemesis or melaena or concealed obstetric/ gynaecological blood loss), hidden sepsis (silent intraperitoneal perforation, early meningococcaemia, or toxic shock syndrome), or a silent cardiovascular event (pulmonary embolus, myocardial infarction).

Most of these conditions, including myocardial infarction without left ventricular failure, will be improved by a fluid challenge.

A fluid challenge, usually $250 \mathrm{ml}$ of crystalloid solution via a wide bore cannula over two minutes, should be given in the first instance. The response to this fluid challenge should be noted and if the patient seems to improve (blood pressure up, heart rate down, peripheral perfusion improved), then fluid loss should be assumed and further fluid should be given.

\section{CASE PROGRESSION}

The patient in the resuscitation room is struggling for breath and is obviously tired. He is given a $250 \mathrm{ml}$ crystalloid fluid challenge over two minutes with a slight improvement in blood pressure, to $90 / 50 \mathrm{~mm} \mathrm{Hg}$, but no change in heart rate or respiratory rate. His peripheral perfusion has improved a little.

A decision is made to proceed with intubation and ventilation, and he undergoes rapid sequence intubation using etomidate, fentanyl, and suxamethonium. He is sedated with low dose infusions of morphine and midazolam, paralysed, and ventilated with low tidal volumes. Haemodynamically he tolerates this reasonably well. Further IV fluids are given and a urinary catheter is inserted to assess ongoing urine output.

Invasive haemodynamic monitoring is started, consisting of a radial arterial line and a triple lumen right internal jugular central venous catheter. Despite 2.5 litres of IV crystalloid, his invasive arterial blood pressure remains at $85 / 45 \mathrm{~mm} \mathrm{Hg}$ and there is clinical evidence of peripheral shut down. His urine output is $10 \mathrm{ml}$ in the first hour.

Chest radiography in the resuscitation room shows a right lower lobe pneumonia and no evidence of pulmonary oedema. A 12 lead ECG shows a sinus tachycardia, Q waves in leads V2, V3, and V4, but no acute changes.

\section{Questions}

- Would you give him further fluid?

- Is it time to start inotropes? If so, which inotropes?

- What other treatments might you consider?

- Where does this man need to be transferred to?

\section{SECONDARY MANAGEMENT}

Many patients will respond to high flow oxygen and IV fluid therapy and peripheral perfusion will improve. Signs of an adequate response to treatment include an improving conscious level, warm peripheries, and reasonable urine output $(>1 \mathrm{ml} / \mathrm{kg} / \mathrm{h})$. Under these circumstances a diagnosis should be sought and the patient should be referred appropriately. There is evidence that patients presenting with haemodynamic instability after trauma have a better outcome if managed in a critical care setting after discharge from the ED. ${ }^{10}$ The same criteria should apply to septic patients who should be managed in a high dependency area.

\section{POOR RESPONSE TO INITIAL TREATMENT}

In severe or ongoing hypovolaemic shock, and in advanced septic shock, initial management may make little or no difference. If the patient's conscious level is deteriorating, haemodynamic parameters worsening, or blood pressures show an increasing metabolic acidosis, then any earlier decision not to ventilate may need urgent review.

If the circulation is still inadequate and there is clinical evidence of shock, it is desirable at this stage to assess the degree of filling of the venous circulation by using a central venous pressure (CVP) monitored fluid challenge.

The response of the CVP to a fluid challenge, $250 \mathrm{ml}$ of IV crystalloid given over two minutes, gives an indication of the compliance of the venous system. If the system remains highly compliant (minimal rise in pressure with further fluid), then further fluid boluses may continue to increase the cardiac output depending on the Starling curve of the right ventricle at that time. If the right heart is becoming non-compliant (that is, significant rise in pressure, greater than $3 \mathrm{~mm} \mathrm{Hg}$ ) with further fluid, it suggests that the systemic venous system is full and that further fluid will simply overload the right ventricle.

It must be remembered that the CVP does not measure the filling pressures on the left side of the heart. This filling pressure has a greater influence on cardiac output and the formation of pulmonary oedema in overload states. This means that CVP monitoring can be misleading. For example, in pulmonary embolism the CVP may be high and show a rise in response to fluid challenge because of outflow obstruction of the right ventricle. Any attempts to treat "fluid overload" (for example, diuretics) would be ineffective and possibly dangerous.

Conversely, in a patient with an extensive anterior myocardial infarction, comparatively normal right ventricular function may occur with a severely impaired left ventricle. The CVP may be low and show a minimal rise in response to a fluid challenge. The left ventricular filling pressures may however be high and further injudicious fluid administration risks the formation of pulmonary oedema.

Despite this, CVP monitoring is simple and ideally used early in the ED setting. Transducer based continuous monitoring is mandatory; manometer systems are inaccurate and cannot be recommended. It is good practice to continue using CVP guided fluid challenges for as long as a clinical response is elicited or until the CVP begins to rise. At this stage, if the patient remains shocked then inotrope therapy should be considered.

In addition to monitoring the CVP, unresponsive shock should prompt a switch to invasive arterial blood pressure monitoring. ${ }^{8}$ Non-invasive monitoring is inaccurate at low pressures and is not sufficiently responsive to guide the minute by minute changes of complex shock management. Furthermore, whether the patient is ventilated or not by this stage, frequent repeated measurements of blood gas pressures will be necessary. It is recommended that the invasive pressures together with continuous ECG monitoring and pulse oximetry are all displayed on one monitor screen simultaneously to permit the integration of all available information.

\section{INOTROPES}

The initiation of inotropic support in the resuscitation room is indicated where shock with or without hypotension is unresponsive to fluid management. This is seen in cardiogenic shock with predominant left ventricular failure or in severe septic shock after CVP guided fluid boluses are producing no further benefit or are giving rise to significant increases in CVP. Inotropes may also be required in the postcardiac arrest setting and in anaphylactic or neurogenic shock that is resistant to fluid therapy.

The goals of inotrope therapy are to raise cardiac output by increasing the heart rate and stroke volume for a given preload and to exert an appropriate effect on the peripheral vascular system. In cardiogenic shock, where the adrenergic 
compensatory mechanisms are usually fully activated and systemic vascular resistance is usually high, the use of inotropes may increase the afterload on the failing left ventricle. This must be weighed against the need to maintain an acceptable mean arterial pressure to permit adequate perfusion of the brain, kidneys, and the heart itself. Furthermore, in an ischaemic failing myocardium, the cost of using inotropes is increased myocardial oxygen consumption and further ischaemia, particularly if the inotrope produces a pronounced tachycardia. ${ }^{8}$

Dobutamine has positive inotropic and chronotropic effects, together with a useful degree of vasodilatation. ${ }^{8}$ Consequently, in severe cardiogenic shock with pulmonary oedema and an adequate systolic blood pressure ( $>90 \mathrm{~mm}$ $\mathrm{Hg}$ ), it is the agent of choice. It must be introduced slowly to avoid tachycardia and arrhythmias. In the presence of an inadequate preload it also commonly causes hypotension.

If hypotension is prominent, especially in the post-arrest situation, adrenaline may be a more appropriate agent. ${ }^{8}$ Despite the hazards of increasing myocardial work, the perfusion of critical organs must be maintained acutely.

In septic and anaphylactic shock, inappropriate vasodilatation and low systemic vascular resistance are the principal problems after fluid resuscitation. ${ }^{8}$ Adrenaline is the drug of choice for patients with anaphylactic shock. ${ }^{7}$ Various vasopressor agents have been used in the treatment of septic shock, including dopamine, adrenaline, noradrenaline, and vasopressin. ${ }^{8}$

There is increasing evidence that noradrenaline may be the agent of choice for patients with severe septic shock. ${ }^{11}$ Noradrenaline has been shown to increase cardiac output, renal blood flow, and urine output when used in septic shock. ${ }^{8}$ As with all inotropes, noradrenaline infusions must be started cautiously and titrated to achieve an adequate mean arterial blood pressure, typically $>65 \mathrm{~mm} \mathrm{Hg}$.

Inotropes should be given via a dedicated lumen through a central venous line. ${ }^{8}$ It is very difficult to titrate doses accurately unless continuous intra-arterial blood pressure monitoring has been established. Any patient requiring inotropes for circulatory support in the ED should be definitively managed in an intensive care setting. ${ }^{8}$

Transfer to the intensive care unit should be carried out with full portable monitoring. It may be more appropriate in certain circumstances to transfer patients directly to the operating room, endoscopy suite, or cardiac catheterisation laboratory for definitive management before intensive care unit admission.

There is some evidence from a single centre study in the US that early goal directed therapy in the ED management of severe sepsis may improve outcome, and this may influence the way that patients with septic shock are treated in the ED in the future. ${ }^{1}$

\section{SPECIFIC TREATMENT OF CAUSES OF SHOCK}

Shock is clearly not a diagnosis in itself. The aetiology of shock should be sought aggressively while the primary and secondary management is carried out to protect and increase tissue perfusion. Some treatable causes of shock are worthy of particular mention. Hypovolaemia attributable to haemorrhage that is continuing requires early definitive surgical management, particularly in trauma. ${ }^{2}{ }^{12-14}$ Ongoing blood loss is usually detected by the failure of fluid boluses to improve the clinical picture in the face of falling or static CVP measurement.

Arrhythmias when present should be considered as potentially contributing to or causing low cardiac output. Bradyarrhythmias may require treatment with atropine or pacing. Tachyarrhythmias, typically atrial fibrillation or flutter, may require cardioversion if there is haemodynamic compromise.

Pulmonary embolism and cardiac tamponade are often suspected when shock is resistant to IV fluid therapy and a high CVP is found. Both conditions can be conveniently differentiated in the resuscitation room by emergency ECG and specific treatment can then be started.

Tension pneumothorax should be identified during the primary assessment of breathing in shock patients, but should be remembered as an important cause of severe circulatory collapse. It may occur immediately after the initiation of mechanical ventilation or shortly after an attempt at central venous cannulation.

Patients with septic shock should be given early and adequate antibiotics in the resuscitation room. ${ }^{15}$ Adequate treatment of sepsis may require urgent surgical intervention to drain abscesses or exteriorise perforated bowel. A brief period of active resuscitation before emergency surgery may be beneficial. ${ }^{16}$

In the absence of any obvious hypovolaemic, cardiac, septic or anaphylactic cause for shock, more unusual causes should be considered. Shock that is resistant to fluid and inotrope therapy may be attributable to an addisonian crisis. If this is a possibility a bolus of IV hydrocortisone should be given promptly after taking a venous blood sample for random cortisol concentrations.

Certain toxins may also provoke cardiovascular collapse but in general the treatment is supportive and cardiovascular management should proceed along similar lines as for sepsis.

Shock in otherwise fit young women should prompt consideration of a diagnosis of toxic shock syndrome and initial investigation should include a digital vaginal and speculum examination.

\section{SUMMARY}

Shock is defined by critical tissue hypoperfusion. It must be rapidly reversed before organ damage is sustained and irreversible. Treatment should therefore begin in the resuscitation room of the ED and should consist of oxygen therapy with or without ventilatory support and a rapid appraisal of the likely causes. Patients with exsanguinating haemorrhage from penetrating torso trauma or ruptured abdominal aortic aneurysms should be transferred to the operating room for definitive management using hypotensive resuscitation.

In all other shock states, except cardiogenic shock with left ventricular failure, intravenous fluids are indicated. Unless this normalises the patient's condition, more invasive investigation and treatment should be started promptly. This should consist of invasive haemodynamic monitoring and repeated IV fluid challenges with CVP guidance.

In shock states unresponsive to intravascular fluid expansion, or in cardiogenic shock where fluid challenges may be hazardous, inotrope therapy is required. The choice of inotrope should be guided by the nature of the problem and the haemodynamic parameters in individual cases. In all shock states reversible factors should be rapidly sought and corrected.

Patients admitted to the ED with all but the most simply managed forms of shock should be transferred to a critical care area once stabilised.

\section{QUESTIONS}

[Answers in the text]

- What is the definition and clinical features of shock?

- What are the hazards of intubation and ventilation in shocked patients and what steps can be taken to minimise them? 
- Explain the utility of a fluid challenge in patients with shock of unclear aetiology.

- Give five reasons for using invasive central venous and arterial monitoring.

- Which inotropes are most useful in the treatment of septic shock?

[Answers in the literature]

- What is the induction agent of choice for intubation in shocked patients?

- What is the evidence for hypotensive resuscitation for penetrating trauma?

- In diabetic ketoacidosis, should unrestricted volumes of fluid be given for shocked patients? What is the recommended maximum? What complications can occur?

- Does goal directed therapy make a difference to the outcome of patients with sepsis?

- What steps would you have to take to allow goal directed therapy of septic shock to occur in your own emergency department?

\section{ACKNOWLEDGEMENTS}

We thank Mr Malcolm Gordon, Dr Phil Munro, and Dr Joan Barber for constructive criticism of earlier versions of the manuscript.

\section{Authors' affiliations}

C A Graham, T R J Parke, Accident and Emergency Department, Southern General Hospital, Glasgow, UK

Funding: none.

Conflicts of interest: none declared.

\section{REFERENCES}

1 Rivers E, Nguyen B, Havstad S, et al. Early goal-directed therapy in the treatment of severe sepsis and septic shock. N Engl J Med 2001;345:1368-77

2 Bickell WH, Wall MJ Jr, Pepe PE, et al. Immediate versus delayed fluid resuscitation for hypotensive patients with penetrating torso injuries. N Engl J Med 1994;331:1105-9.

3 Chesnut RM. The management of severe traumatic brain injury. Emerg Med Clin North Am 1997; 15:581-604.

4 Chesnut RM. Avoidance of hypotension: conditio sine qua non of successful severe head-injury management. J Trauma 1997;42(suppl 9):4-9s.

5 Brown AF. Therapeutic controversies in the management of acute anaphylaxis. J Accid Emerg Med 1998;15:89-95.

6 Brown AF, McKinnon D, Chu K. Emergency department anaphylaxis: a review of 142 patients in a single year. J Allerg Clin Immunol 2001; 108:861-6.

7 Brown AF. Anaphylaxis: quintessence, quarrels, and quandaries. Emerg Med J $2001 ; 18: 328$.

8 Anonymous. Practice parameters for hemodynamic support of sepsis in adult patients in sepsis. Task Force of the American College of Critical Care Medicine, Society of Critical Care Medicine. Crit Care Med 1999;27:639-60.

9 Levy MM, Fink MP, Marshall JC, et al. 2001 SCCM/ESICM/ACCP/ATS/SIS international sepsis definitions conference. Intensive Care Med 2003;29:530-8.

10 Parke TR, Henry J, Grant PT, et al. Increased survival after serious injury in patients admitted directly to critical care areas from the accident and emergency department. Injury 1998;29:697-703.

11 Martin C, Papazian L, Perrin G, et al. Norepinephrine or dopamine for the treatment of hyperdynamic septic shock? Chest 1993;103:1826-31.

12 Giannoudis PV. Current concepts of the inflammatory response after major trauma: an update. Injury 2003;34:397-404.

13 Johnson JW, Gracias VH, Schwab CW, et al. Evolution in damage control for exsanguinating penetrating abdominal injury. J Trauma 2001;51:261-9.

14 Wilson M, Davis DP, Coimbra R. Diagnosis and monitoring of hemorrhagic shock during the initial resuscitation of multiple trauma patients: a review. J Emerg Med 2003;24:413-22.

15 Kollef MH, Sherman G, Ward S, et al. Inadequate antimicrobial treatment of infections: a risk factor for hospital mortality among critically ill patients. Chest 1999; 115:462-74.

16 MacKenzie IM. The haemodynamics of human septic shock. Anaesthesia 2001;56:130-44 